\title{
Entire Functions and Their Derivatives Share Two Finite Sets
}

\author{
Chao MenG* \\ Department of Mathematics, Shandong University, Jinan 250100, P. R. China \\ e-mail : mengchaosdu@yahoo.com.cn and mengchao@mail.sdu.edu.cn \\ PeI-Chu Hu \\ Department of Mathematics, Shandong University, Jinan 250100, P. R. China \\ e-mail : pchu@sdu.edu.cn
}

Abstract. In this paper, we study the uniqueness of entire functions and prove the following theorem. Let $n(\geq 5), k$ be positive integers, and let $S_{1}=\left\{z: z^{n}=1\right\}$, $S_{2}=\left\{a_{1}, a_{2}, \cdots, a_{m}\right\}$, where $a_{1}, a_{2}, \cdots, a_{m}$ are distinct nonzero constants. If two nonconstant entire functions $f$ and $g$ satisfy $E_{f}\left(S_{1}, 2\right)=E_{g}\left(S_{1}, 2\right)$ and $E_{f(k)}\left(S_{2}, \infty\right)=$ $E_{g^{(k)}}\left(S_{2}, \infty\right)$, then one of the following cases must occur: (1) $f=t g,\left\{a_{1}, a_{2}, \cdots, a_{m}\right\}=$ $t\left\{a_{1}, a_{2}, \cdots, a_{m}\right\}$, where $t$ is a constant satisfying $t^{n}=1$; (2) $f(z)=d e^{c z}, g(z)=\frac{t}{d} e^{-c z}$, $\left\{a_{1}, a_{2}, \cdots, a_{m}\right\}=(-1)^{k} c^{2 k} t\left\{\frac{1}{a_{1}}, \cdots, \frac{1}{a_{m}}\right\}$, where $t, c, d$ are nonzero constants and $t^{n}=1$. The results in this paper improve the result given by Fang (M.L. Fang, Entire functions and their derivatives share two finite sets, Bull. Malaysian Math. Sc. Soc. 24(2001), $7-16)$.

\section{Introduction, definitions and results}

Let $f$ and $g$ be two nonconstant meromorphic functions defined in the open complex plane $C$. If for some $a \in C \cup\{\infty\}, f$ and $g$ have the same set of $a$-points with the same multiplicities then we say that $f$ and $g$ share the value $a$ CM (counting multiplicities). If we do not take the multiplicities into account, $f$ and $g$ are said to share the value $a$ IM (ignoring multiplicities). We assume that the reader is familiar with the notations of Nevanlinna theory that can be found, for instance, in [5] or [9].

Let $S$ be a set of distinct elements of $C \cup\{\infty\}$ and $E_{f}(S)=\cup_{a \in S}\{z: f(z)-a=$ $0\}$, where each zero is counted according to its multiplicity. If we do not count the multiplicity the set $\cup_{a \in S}\{z: f(z)-a=0\}$ is denoted by $\bar{E}_{f}(S)$. If $E_{f}(S)=E_{g}(S)$ we say that $f$ and $g$ share the set $S$ CM. On the other hand, if $\bar{E}_{f}(S)=\bar{E}_{g}(S)$, we say that $f$ and $g$ share the set $S$ IM. Let $m$ be a positive integer or infinity and $a \in C \cup\{\infty\}$. We denote by $E_{m)}(a, f)$ the set of all $a$-points of $f$ with multiplicities not exceeding $m$, where an $a$-point is counted according to its multiplicity. For a

* Corresponding author.

Received March 23, 2008; accepted May 16, 2008.

2000 Mathematics Subject Classification: 30D35.

Key words and phrases: entire function, share set, uniqueness. 
set $S$ of distinct elements of $C$ we define $E_{m)}(S, f)=\cup_{a \in S} E_{m)}(a, f)$. If for some $a \in C \cup\{\infty\}, E_{\infty)}(a, f)=E_{\infty)}(a, g)$, we say that $f$ and $g$ share the value $a \mathrm{CM}$. We can define $\bar{E}_{m)}(a, f)$ and $\bar{E}_{m)}(S, f)$ similarly.

In 1977, Gross [4]posed the following question.

Question. Can one find two finite sets $S_{j}(j=1,2)$ such that any two nonconstant entire functions $f$ and $g$ satisfying $E_{f}\left(S_{j}\right)=E_{g}\left(S_{j}\right)$ for $j=1,2$ must be identical ?

$\mathrm{Yi}[10]$ gave a positive answer to the question. He proved.

Theorem $\mathbf{A}([\mathbf{1 0}])$. Let $f$ and $g$ be two nonconstant entire functions, $n \geq 5 a$ positive integer, and let $S_{1}=\left\{z: z^{n}=1\right\}, S_{2}=\{a\}$, where $a \neq 0$ is a constant satisfying $a^{2 n} \neq 1$. If $E_{f}\left(S_{j}\right)=E_{g}\left(S_{j}\right)$ for $j=1,2$, then $f \equiv g$.

In 2001, Fang [3] investigated the question and proved the following theorems.

Theorem $\mathbf{B}([3])$. Let $f$ and $g$ be two nonconstant entire functions, $n \geq 5, k$ two positive integers, and let $S_{1}=\left\{z: z^{n}=1\right\}, S_{2}=\{a, b, c\}$, where $a, b, c$ are nonzero finite distinct constants satisfying $a^{2} \neq b c, b^{2} \neq a c, c^{2} \neq a b$. If $E_{f}\left(S_{1}\right)=E_{g}\left(S_{1}\right)$ and $E_{f^{(k)}}\left(S_{2}\right)=E_{g^{(k)}}\left(S_{2}\right)$, then $f \equiv g$.

Theorem $\mathbf{C}([3])$. Let $f$ and $g$ be two nonconstant entire functions, $n \geq 5, k$ two positive integers, and let $S_{1}=\left\{z: z^{n}=1\right\}, S_{2}=\{a, b\}$, where $a, b$ are two nonzero finite distinct constants. If $E_{f}\left(S_{1}\right)=E_{g}\left(S_{1}\right)$ and $E_{f^{(k)}}\left(S_{2}\right)=E_{g^{(k)}}\left(S_{2}\right)$, then one of the following cases must occur: (1) $f \equiv g ;(2) b=-a, f=e^{c z+d}$, $g=t e^{-c z-d}$, where $c, d$, $t$ are three constants satisfying $t^{n}=1$ and $(-1)^{k} t c^{2 k}=a^{2}$; (3) $f=e^{c z+d}, g=t e^{-c z-d}$, where $c, d$, $t$ are three constants satisfying $t^{n}=1$ and $(-1)^{k} t c^{2 k}=a b ;(4) b=-a, f \equiv-g$.

Theorem $\mathbf{D}([\mathbf{3}])$. Let $f$ and $g$ be two nonconstant entire functions, $n \geq 5, k$ two positive integers, and let $S_{1}=\left\{z: z^{n}=1\right\}, S_{2}=\{a\}$, where $a \neq 0, \infty$. If $E_{f}\left(S_{1}\right)=E_{g}\left(S_{1}\right)$ and $E_{f(k)}\left(S_{2}\right)=E_{g^{(k)}}\left(S_{2}\right)$, then one of the following cases must occur: (1) $f \equiv g$; (2) $f=e^{c z+d}, g=t e^{-c z-d}$, where $c, d$, $t$ are three constants satisfying $t^{n}=1$ and $(-1)^{k} t c^{2 k}=a^{2}$.

In this paper, we consider the more general sets $S_{1}=\left\{z: z^{n}=1\right\}, S_{2}=$ $\left\{a_{1}, a_{2}, \cdots, a_{m}\right\}$, where $a_{1}, a_{2}, \cdots, a_{m}$ are distinct nonzero constants. To state the main results of this paper, we require the following notion of weighted sharing which was introduced by I. Lahiri [6], [7].

Definition 1([6]). For a complex number $a \in C \cup\{\infty\}$, we denote by $E_{k}(a, f)$ the set of all $a$-points of $f$ where an $a$-point with mutiplicity $m$ is counted $m$ times if $m \leq k$ and $k+1$ times if $m>k$. For a complex number $a \in C \cup\{\infty\}$, such that $E_{k}(a, f)=E_{k}(a, g)$, then we say that $f$ and $g$ share the value $a$ with weight $k$.

The definition implies that if $f, g$ share a value $a$ with weight $k$ then $z_{0}$ is a zero of $f-a$ with multiplicity $m(\leq k)$ if and only if it is a zero of $g-a$ with multiplicity 
$m(\leq k)$ and $z_{0}$ is a zero of $f-a$ with multiplicity $m(>k)$ if and only if it is a zero of $g-a$ with multiplicity $n(>k)$, where $m$ is not necessarily equal to $n$. We write $f, g$ share $(a, k)$ to mean that $f, g$ share the value $a$ with weight $k$. Clearly if $f, g$ share $(a, k)$ then $f, g$ share $(a, p)$ for all integer $p, 0 \leq p<k$. Also we note that $f$, $g$ share a value $a$ IM or CM if and only if $f, g$ share $(a, 0)$ or $(a, \infty)$ respectively.

Definition 2([6]). Let $S$ be a set of distinct elements of $C \cup\{\infty\}$ and $k$ a nonnegative integer or $\infty$. We denote by $E_{f}(S, k)$ the set $\cup_{a \in S} E_{k}(a, f)$. Clearly $E_{f}(S)=E_{f}(S, \infty)$ and $\bar{E}_{f}(S)=E_{f}(S, 0)$.

With the notion of weighted sharing of sets we prove the following results which improve Theorem B, Theorem C and Theorem D.

Theorem 1. Let $n(\geq 5), k$ be positive integers, and let $S_{1}=\left\{z: z^{n}=1\right\}$, $S_{2}=\left\{a_{1}, a_{2}, \cdots, a_{m}\right\}$, where $a_{1}, a_{2}, \cdots, a_{m}$ are distinct nonzero constants. If two nonconstant entire functions $f$ and $g$ satisfy $E_{f}\left(S_{1}, 2\right)=E_{g}\left(S_{1}, 2\right)$ and $E_{f^{(k)}}\left(S_{2}, \infty\right)=E_{g^{(k)}}\left(S_{2}, \infty\right)$, then one of the following cases must occur: (1) $f=t g$, $\left\{a_{1}, a_{2}, \cdots, a_{m}\right\}=t\left\{a_{1}, a_{2}, \cdots, a_{m}\right\}$, where $t$ is a constant satisfying $t^{n}=1$; (2) $f(z)=d e^{c z}, g(z)=\frac{t}{d} e^{-c z},\left\{a_{1}, a_{2}, \cdots, a_{m}\right\}=(-1)^{k} c^{2 k} t\left\{\frac{1}{a_{1}}, \cdots, \frac{1}{a_{m}}\right\}$, where $t, c$, $d$ are nonzero constants and $t^{n}=1$.

Theorem 2. Let $n(\geq 5), k$ be positive integers, and let $S_{1}=\left\{z: z^{n}=1\right\}$, $S_{2}=\left\{a_{1}, a_{2}, \cdots, a_{m}\right\}$, where $a_{1}, a_{2}, \cdots, a_{m}$ are distinct nonzero constants. If two nonconstant entire functions $f$ and $g$ satisfy $E_{f}\left(S_{1}, 1\right)=E_{g}\left(S_{1}, 1\right)$ and $E_{f^{(k)}}\left(S_{2}, \infty\right)=E_{g^{(k)}}\left(S_{2}, \infty\right)$, then one of the following cases must occur: (1) $f=t g$, $\left\{a_{1}, a_{2}, \cdots, a_{m}\right\}=t\left\{a_{1}, a_{2}, \cdots, a_{m}\right\}$, where $t$ is a constant satisfying $t^{n}=1 ;(2)$ $f(z)=d e^{c z}, g(z)=\frac{t}{d} e^{-c z},\left\{a_{1}, a_{2}, \cdots, a_{m}\right\}=(-1)^{k} c^{2 k} t\left\{\frac{1}{a_{1}}, \cdots, \frac{1}{a_{m}}\right\}$, where $t, c$, $d$ are nonzero constants and $t^{n}=1$.

Theorem 3. Let $n(\geq 8), k$ be positive integers, and let $S_{1}=\left\{z: z^{n}=1\right\}$, $S_{2}=\left\{a_{1}, a_{2}, \cdots, a_{m}\right\}$, where $a_{1}, a_{2}, \cdots, a_{m}$ are distinct nonzero constants. If two nonconstant entire functions $f$ and $g$ satisfy $E_{f}\left(S_{1}, 0\right)=E_{g}\left(S_{1}, 0\right)$ and $E_{f^{(k)}}\left(S_{2}, \infty\right)=E_{g^{(k)}}\left(S_{2}, \infty\right)$, then one of the following cases must occur: (1) $f=t g$, $\left\{a_{1}, a_{2}, \cdots, a_{m}\right\}=t\left\{a_{1}, a_{2}, \cdots, a_{m}\right\}$, where $t$ is a constant satisfying $t^{n}=1 ;$ (2) $f(z)=d e^{c z}, g(z)=\frac{t}{d} e^{-c z},\left\{a_{1}, a_{2}, \cdots, a_{m}\right\}=(-1)^{k} c^{2 k} t\left\{\frac{1}{a_{1}}, \cdots, \frac{1}{a_{m}}\right\}$, where $t, c$, $d$ are nonzero constants and $t^{n}=1$.

Without the notion of weighted sharing of sets we prove the following theorem which also improves Theorem B, Theorem C and Theorem D.

Theorem 4. Let $n(\geq 5), k$ be positive integers, and let $S_{1}=\left\{z: z^{n}=1\right\}$, $S_{2}=\left\{a_{1}, a_{2}, \cdots, a_{m}\right\}$, where $a_{1}, a_{2}, \cdots, a_{m}$ are distinct nonzero constants. If two nonconstant entire functions $f$ and $g$ satisfy $\bar{E}_{4)}\left(S_{1}, f\right)=\bar{E}_{4)}\left(S_{1}, g\right), E_{2)}\left(S_{1}, f\right)=$ $E_{2)}\left(S_{1}, g\right)$ and $E_{f(k)}\left(S_{2}, \infty\right)=E_{g^{(k)}}\left(S_{2}, \infty\right)$, then one of the following cases must occur: (1) $f=t g,\left\{a_{1}, a_{2}, \cdots, a_{m}\right\}=t\left\{a_{1}, a_{2}, \cdots, a_{m}\right\}$, where $t$ is a con- 
stant satisfying $t^{n}=1 ;$ (2) $f(z)=d e^{c z}, g(z)=\frac{t}{d} e^{-c z},\left\{a_{1}, a_{2}, \cdots, a_{m}\right\}=$ $(-1)^{k} c^{2 k} t\left\{\frac{1}{a_{1}}, \cdots, \frac{1}{a_{m}}\right\}$, where $t, c, d$ are nonzero constants and $t^{n}=1$.

\section{Some lemmas}

In this section, we present some lemmas which will be needed in the sequel. We will denote by $H$ the following function:

$$
H=\left(\frac{F^{\prime \prime}}{F^{\prime}}-\frac{2 F^{\prime}}{F-1}\right)-\left(\frac{G^{\prime \prime}}{G^{\prime}}-\frac{2 G^{\prime}}{G-1}\right)
$$

Lemma 1([8]). Let $f$ be a nonconstant meromorphic function, and let $a_{0}, a_{1}, a_{2}, \cdots, a_{n}$ be finite complex numbers, $a_{n} \neq 0$. Then

$$
T\left(r, a_{n} f^{n}+\cdots+a_{2} f^{2}+a_{1} f+a_{0}\right)=n T(r, f)+S(r, f) .
$$

Lemma 2([7]). Let $H$ be defined as above. If $F$ and $G$ share $(1,2)$ and $H \not \equiv 0$, then

$$
T(r, F) \leq N_{2}\left(r, \frac{1}{F}\right)+N_{2}\left(r, \frac{1}{G}\right)+N_{2}(r, F)+N_{2}(r, G)+S(r, F)+S(r, G),
$$

the same inequality holds for $T(r, G)$.

Lemma 3([2]). Let $H$ be defined as above. If $F$ and $G$ share $(1,1)$ and $H \not \equiv 0$, then

$$
\begin{aligned}
T(r, F) \leq & N_{2}\left(r, \frac{1}{F}\right)+N_{2}(r, F)+N_{2}\left(r, \frac{1}{G}\right)+N_{2}(r, G) \\
& +\frac{1}{2} \bar{N}\left(r, \frac{1}{F}\right)+\frac{1}{2} \bar{N}(r, F)+S(r, F)+S(r, G),
\end{aligned}
$$

the same inequality holds for $T(r, G)$.

Lemma 4([11]). Let $H$ be defined as above. If $H \equiv 0$ and

$$
\limsup _{r \rightarrow \infty} \frac{\bar{N}\left(r, \frac{1}{F}\right)+\bar{N}\left(r, \frac{1}{G}\right)+\bar{N}(r, F)+\bar{N}(r, G)}{T(r)}<1, r \in I,
$$

where $I$ is a set with infinite linear measure and $T(r)=\max \{T(r, F), T(r, G)\}$, then $F G \equiv 1$ or $F \equiv G$.

Lemma 5([2]). Let $F, G$ be two nonconstant meromorphic functions such that 
they share $(1,0)$, and $H \not \equiv 0$. Then

$$
\begin{array}{r}
T(r, F) \leq N_{2}\left(r, \frac{1}{F}\right)+N_{2}(r, F)+N_{2}\left(r, \frac{1}{G}\right)+N_{2}(r, G)+2 \bar{N}\left(r, \frac{1}{F}\right)+2 \bar{N}(r, F) \\
+\bar{N}\left(r, \frac{1}{G}\right)+\bar{N}(r, G)+S(r, F)+S(r, G),
\end{array}
$$

the same inequality holds for $T(r, G)$.

Lemma 6([1]). Let $F, G$ be two nonconstant meromorphic functions such that $\bar{E}_{4)}(1, F)=\bar{E}_{4)}(1, G)$ and $E_{2)}(1, F)=E_{2}(1, G)$, then one of the following cases holds $(1) T(r, F)+T(r, G) \leq 2\left\{N_{2}\left(r, \frac{1}{F}\right)+N_{2}\left(r, \frac{1}{G}\right)+N_{2}(r, F)+N_{2}(r, G)\right\}+S(r, F)+$ $S(r, G) ;(2) F \equiv G ;(3) F G \equiv 1$.

Lemma $\mathbf{7}([5])$. Let $f$ be a nonconstant meromorphic function, $n$ be a positive integer, and let $\Psi$ be a function of the form $\Psi=f^{n}+Q$, where $Q$ is a differential polynomial of $f$ with degree $\leq n-1$. If

$$
N(r, f)+N\left(r, \frac{1}{\Psi}\right)=S(r, f)
$$

then $\Psi=(f+\alpha)^{n}$, where $\alpha$ is a meromorphic function with $T(r, \alpha)=S(r, f)$, determined by the term of degree $n-1$ in $Q$.

\section{Proof of theorem 1}

Set $F=f^{n}, G=g^{n}$. From $E_{f}\left(S_{1}, 2\right)=E_{g}\left(S_{1}, 2\right)$, we deduce $F$ and $G$ share $(1,2)$. By Lemma 1 , we have

$$
T(r, F)=n T(r, f)+S(r, f), \quad T(r, G)=n T(r, g)+S(r, g) .
$$

Assume $H \not \equiv 0$. By Lemma 2, we have

$$
\begin{aligned}
T(r, F) & =n T(r, f)+S(r, f) \\
& \leq N_{2}\left(r, \frac{1}{F}\right)+N_{2}\left(r, \frac{1}{G}\right)+S(r, F)+S(r, G) \\
& \leq 2 T(r, f)+2 T(r, g)+S(r, f)+S(r, g) .
\end{aligned}
$$

Similarly, we have

$$
\begin{aligned}
T(r, G) & =n T(r, g)+S(r, f) \\
& \leq N_{2}\left(r, \frac{1}{F}\right)+N_{2}\left(r, \frac{1}{G}\right)+S(r, F)+S(r, G) \\
& \leq 2 T(r, f)+2 T(r, g)+S(r, f)+S(r, g) .
\end{aligned}
$$

Combining (2) and (3) together we have

$$
(n-4) T(r, f)+(n-4) T(r, g) \leq S(r, f)+S(r, g),
$$


which contradicts $n \geq 5$. Thus $H \equiv 0$. By Lemma 4 , we have $F G \equiv 1$ or $F \equiv G$, that is $f=t g$ or $f g=t$ where $t$ is a constant and $t^{n}=1$. Next we consider the following two cases:

Case 1. $f=t g$. Then $f^{(k)}=t g^{(k)}$. By $E_{f^{(k)}}\left(S_{2}, \infty\right)=E_{g^{(k)}}\left(S_{2}, \infty\right)$, we get $\left\{a_{1}, a_{2}, \cdots, a_{m}\right\}=t\left\{a_{1}, a_{2}, \cdots, a_{m}\right\}$.

Case 2. $f g=t$. Then there exists an entire function $h$ such that $f=e^{h}$ and $g=t e^{-h}$. Therefore

$$
f^{(i)}=\alpha_{i} f, g^{(i)}=\beta_{i} g, i=1,2, \cdots,
$$

where $\alpha_{1}=h^{\prime}, \beta_{1}=-h^{\prime}$, and $\alpha_{i}, \beta_{i}$ satisfy the following recurrence formulas, respectively.

$$
\alpha_{i+1}=\alpha_{i}^{\prime}+\alpha_{i}^{2}, \beta_{i+1}=\beta_{i}^{\prime}+\beta_{i}^{2}, i=1,2, \cdots,
$$

Without loss of the generality, we assume that $a_{1}$ is not an exceptional value of $f^{(k)}$. Suppose $f^{(k)}\left(z_{0}\right)=a_{1}$. Then $\frac{t}{a_{1}} \alpha_{k}\left(z_{0}\right) \beta_{k}\left(z_{0}\right)=g^{(k)}\left(z_{0}\right) \in S_{2}$. Therefore,

$$
\prod_{j=1}^{m}\left(\frac{t}{a_{1}} \alpha_{k}\left(z_{0}\right) \beta_{k}\left(z_{0}\right)-a_{j}\right)=0 .
$$

Note that $\bar{N}\left(r, 1 /\left(f^{(k)}-a_{1}\right)\right) \neq S(r, f)$. We get

$$
\prod_{j=1}^{m}\left(\frac{t}{a_{1}} \alpha_{k} \beta_{k}-a_{j}\right)=0
$$

which implies that $\alpha_{k} \beta_{k}$ is a nonzero constant. And thus $\alpha_{k}$ and $\beta_{k}$ have no zeros. The recurrence formulas in (6) show that

$$
\alpha_{k}=\alpha_{1}^{k}+P\left(\alpha_{1}\right), \beta_{k}=\beta_{1}^{k}+Q\left(\beta_{1}\right),
$$

where $P\left(\alpha_{1}\right)$ is a differential polynomial in $\alpha_{1}$ of degree $k-1$, and $Q\left(\beta_{1}\right)$ is a differential polynomial in $\beta_{1}$ of degree $k-1$. If $\alpha_{1}$ and $\beta_{1}$ are not constants, then by Lemma 7, we have

$$
\alpha_{k}=\left(\alpha_{1}+\frac{\gamma_{1}}{k}\right)^{k}, \beta_{k}=\left(\beta_{1}+\frac{\gamma_{2}}{k}\right)^{k},
$$

where $\gamma_{1}, \gamma_{2}$ are small functions of $\alpha_{1}$ and $\beta_{1}$, respectively. Note that $\alpha_{1}=-\beta_{1}=$ $h^{\prime}$. We conclude that $\alpha_{k} \beta_{k}$ can not be constant, which is a contradiction. Hence one of $\alpha_{1}$ and $\beta_{1}$ is constant. Thus $h$ is a linear function. Therefore, $f(z)=d e^{c z}$ and $g(z)=\frac{t}{d} e^{-c z}$, where $c, d$ are nonzero constants. Now from $E_{f^{(k)}}\left(S_{2}, \infty\right)=$ 
$E_{g^{(k)}}\left(S_{2}, \infty\right)$, we get $\left\{a_{1}, a_{2}, \cdots, a_{m}\right\}=(-1)^{k} c^{2 k} t\left\{\frac{1}{a_{1}}, \cdots, \frac{1}{a_{m}}\right\}$, which completes the proof of Theorem 1 .

\section{Proof of theorem 2}

Set $F=f^{n}, G=g^{n}$. From $E_{f}\left(S_{1}, 1\right)=E_{g}\left(S_{1}, 1\right)$, we deduce $F$ and $G$ share $(1,1)$. By Lemma 1 , we have

$$
T(r, F)=n T(r, f)+S(r, f), \quad T(r, G)=n T(r, g)+S(r, g) .
$$

Assume $H \not \equiv 0$. By Lemma 3, we have

$$
\begin{aligned}
T(r, F) & =n T(r, f)+S(r, f) \\
& \leq N_{2}\left(r, \frac{1}{F}\right)+N_{2}\left(r, \frac{1}{G}\right)+\frac{1}{2} \bar{N}\left(r, \frac{1}{F}\right)+S(r, F)+S(r, G) \\
& \leq \frac{5}{2} T(r, f)+2 T(r, g)+S(r, f)+S(r, g) .
\end{aligned}
$$

Similarly, we have

$$
\begin{aligned}
T(r, G) & =n T(r, g)+S(r, g) \\
& \leq N_{2}\left(r, \frac{1}{F}\right)+N_{2}\left(r, \frac{1}{G}\right)+\frac{1}{2} \bar{N}\left(r, \frac{1}{G}\right)+S(r, F)+S(r, G) \\
& \leq 2 T(r, f)+\frac{5}{2} T(r, g)+S(r, f)+S(r, g) .
\end{aligned}
$$

Combining (12) and (13) together we have

$$
\left(n-\frac{9}{2}\right) T(r, f)+\left(n-\frac{9}{2}\right) T(r, g) \leq S(r, f)+S(r, g),
$$

which contradicts $n \geq 5$. Thus $H \equiv 0$. By Lemma 4 , we have $F G \equiv 1$ or $F \equiv G$, that is $f=t g$ or $f g=t$ where $t$ is a constant and $t^{n}=1$. Proceeding as in the proof of Theorem 1, we get the conclusion of Theorem 2. This completes the proof of Theorem 2.

\section{Proof of theorem 3}

Set $F=f^{n}, G=g^{n}$. From $E_{f}\left(S_{1}, 0\right)=E_{g}\left(S_{1}, 0\right)$, we deduce $F$ and $G$ share $(1,0)$. By Lemma 1 , we have

$$
T(r, F)=n T(r, f)+S(r, f), \quad T(r, G)=n T(r, g)+S(r, g) .
$$


Assume $H \not \equiv 0$. By Lemma 5, we have

$$
\begin{aligned}
T(r, F) & =n T(r, f)+S(r, f) \\
& \leq N_{2}\left(r, \frac{1}{F}\right)+N_{2}\left(r, \frac{1}{G}\right)+2 \bar{N}\left(r, \frac{1}{F}\right)+\bar{N}\left(r, \frac{1}{G}\right)+S(r, F)+S(r, G) \\
& \leq 4 T(r, f)+3 T(r, g)+S(r, f)+S(r, g) .
\end{aligned}
$$

Similarly, we have

$$
\begin{aligned}
T(r, G) & =n T(r, g)+S(r, g) \\
& \leq 3 T(r, f)+4 T(r, g)+S(r, f)+S(r, g) .
\end{aligned}
$$

Combining (16) and (17) together we have

$$
(n-7) T(r, f)+(n-7) T(r, g) \leq S(r, f)+S(r, g),
$$

which contradicts $n \geq 8$. Thus $H \equiv 0$. By Lemma 4 , we have $F G \equiv 1$ or $F \equiv G$, that is $f=t g$ or $f g=t$ where $t$ is a constant and $t^{n}=1$. Proceeding as in the proof of Theorem 1, we get the conclusion of Theorem 3. This completes the proof of Theorem 3.

\section{Proof of theorem 4}

Set $F=f^{n}, G=g^{n}$. By Lemma 1, we have

$$
T(r, F)=n T(r, f)+S(r, f), \quad T(r, G)=n T(r, g)+S(r, g) .
$$

From $\bar{E}_{4)}\left(S_{1}, f\right)=\bar{E}_{4)}\left(S_{1}, g\right), E_{2)}\left(S_{1}, f\right)=E_{2)}\left(S_{1}, g\right)$, we deduce $\bar{E}_{4)}(1, F)=$ $\bar{E}_{4)}(1, G), E_{2)}(1, F)=E_{2)}(1, G)$. Then $F$ and $G$ satisfy the condition of Lemma 6 . We assume Case (1) in Lemma 6 holds, that is,

$$
\begin{aligned}
T(r, F)+T(r, G) & \leq 2\left\{N_{2}\left(r, \frac{1}{F}\right)+N_{2}\left(r, \frac{1}{G}\right)\right\}+S(r, F)+S(r, G) \\
& \leq 4 T(r, f)+4 T(r, g)+S(r, f)+S(r, g) .
\end{aligned}
$$

Combining (19) and (20) together we have

$$
(n-4) T(r, f)+(n-4) T(r, g) \leq S(r, f)+S(r, g),
$$

which contradicts $n \geq 5$. Thus by Lemma 6 , we get $F \equiv G$ or $F G \equiv 1$, that is, $f=t g$ or $f g=t$ where $t$ is a constant and $t^{n}=1$. Proceeding as in the proof of Theorem 1, we get the conclusion of Theorem 4. This completes the proof of Theorem 4. 


\section{References}

[1] A. Banerjee, On uniqueness of meromorphic functions when two differential monomials share one value, Bull. Korean Math. Soc., 44(2007), 607-622.

[2] A. Banerjee, Meromorphic functions sharing one value, Int. J. Math. Math. Sci., 22(2005), 3587-3598.

[3] M. L. Fang, Entire functions and their derivatives share two finite sets, Bull. Malaysian Math. Sc. Soc., 24(2001), 7-16.

[4] F. Gross, Factorization of meromorphic functions and some open problems, Lecture Notes in Math. 599, Springer, Berlin, 1977.

[5] W. K. Hayman, Meromorphic Functions, Clarendon, Oxford, 1964

[6] I. Lahiri, Weighted sharing and uniqueness of meromorphic functions, Nagoya Math. J., 161(2001), 193-206.

[7] I. Lahiri, Weighted value sharing and uniqueness of meromorphic functions, Complex Variables Theory Appl., 46(2001), 241-253.

[8] C. C. Yang, On deficiencies of differential polynomials, Math. Z., 125(1972), 107-112.

[9] L. Yang, Value Distribution Theory, Springer-Verlag, Berlin, 1993.

[10] H. X. Yi, On the uniqueness of meromorphic functions and a problem of Gross, Science in China, 24(1994), 457-466.

[11] H. X. Yi, Meromorphic functions that share one or two values, Complex Variables Theory Appl., 28(1995), 1-11. 